\title{
THE SANDERS-KOITER SHELL EQUATIONS CAN BE REDUCED TO TWO COUPLED EQUATIONS FOR ALL MINIMAL MIDSURFACES*
}

\author{
$\mathrm{By}$ \\ G. E. LATTA AND J. G. SIMMONDS \\ University of Virginia
}

\begin{abstract}
For an elastically isotropic shell of constant thickness with an analytic midsurface of zero mean curvature, it is shown that the linear Sanders-Koiter equations can be reduced exactly to two coupled fourth-order partial differential equations, involving as unknowns a stress function and the rotation of the midsurface about the normal.

1. Introduction. The partial differential equations of classical, linear shell theory are elliptic and of eight order. These equations may take a variety of forms depending on the choice of the dependent variables, but for shells subject to static edge loads only, the static-geometric analogy suggests that dual pairs of dependent variables ought to be chosen.

A fundamental problem in linear shell theory is to reduce the governing equations to two coupled fourth-order equations. Such a reduced system can be expected to be immensely more amenable to analysis than its parent, especially with regard to asymptotic techniques. For example, in the restricted theory of shallow shells, the usefulness of working with just two coupled equations has been long recognized.

In the general theory of shells, it has been known that the equations for shells of revolution [1, 2], general cylindrical shells [1], and catenoidal and helicoidal shells [3] can be reduced to two coupled fourth-order equations. These reductions employ various pairs of dependent variables, but a reduction which works with one pair of unknowns will not necessarily go through with another. In the present paper we show that the method of reduction developed in [3] for catenoidal and helicoidal shells may be generalized to any shell with an analytic, minimal midsurface, excluding a plane. The dependent variables involved are a stress function and the rotation of the midsurface displacement field about the normal. This is in contrast to the reductions in $[1,2]$ which use either the normal components of the midsurface displacement and stress function vectors or the linear invariants of the stress resultant and bending strain tensor.
\end{abstract}

2. The governing equations. In [3] it was shown that the linear Sanders-Koiter equations, for an elastically isotropic shell of constant thickness with a minimal midsurface, could be reduced to the form

$$
\begin{aligned}
& S=L(F)-D W(T), \\
& T=L(G)+A W(S),
\end{aligned}
$$

* Received December 28, 1973. This research was supported by the Office of Naval Reseach under contract N00014-69-A-0060-0010 and the National Science Foundation under Grant GP-38197. 


$$
\begin{aligned}
& W(F)+D L(T)+D(1-\mu)\left(\nabla^{2}-2 K\right) G=0 \\
& W(G)-A L(S)-A(1+\nu)\left(\nabla^{2}-2 K\right) F=0
\end{aligned}
$$

In these equations $A, D, \mu$, and $\nu$ are elastic constants and

$$
\begin{aligned}
L(F) & =\epsilon^{\alpha \gamma}\left(K^{-1} b_{\alpha}{ }^{\beta} F_{\mid \beta}\right)_{\mid \gamma}, \\
W(F) & =-\left(K^{-1} b^{\alpha \beta} F_{\mid \alpha}\right)_{\mid \beta}, \\
\nabla^{2}(F) & =a^{\alpha \beta} F_{\mid \alpha \beta},
\end{aligned}
$$

where $\epsilon^{\alpha \gamma}$ is the contravariant midsurface permutation tensor, $K$ is the (negative) Gaussian curvature, $b_{\alpha}{ }^{\beta}=a^{\beta \gamma} b_{\gamma \alpha}, b^{\alpha \beta}=a^{\alpha \gamma} b_{\gamma}{ }^{\beta}, a^{\alpha \gamma} a_{\gamma \beta}=\delta_{\beta}{ }^{\alpha}$, the Kronecker delta, and $a_{\alpha \beta}$ and $b_{\alpha \beta}$ are, respectively, the covariant metric and curvature tensors of the midsurface. A vertical bar, followed by an $\alpha$, denotes covariant differentiation with respect to the surface coordinate $u^{\alpha}$. The unknowns $F, G, S$, and $T$ represent, respectively, a stress function, the rotation of the midsurface displacement about the midsurface normal, and the linear invariants of the stress resultant and bending strain tensors.

The derivation of (2.1)-(2.4) from the Sanders-Koiter equations is exact, but does require the use of (weakly) coupled stress-strain relations. The relative differences between the stress and displacement fields associated with these relations and those corresponding to the conventional uncoupled stress-strain relations have been shown to be negligible in a mean-square sense, being of the same order of magnitude as the errors inherent in the Sanders-Koiter theory itself [4].

Our aim in the present paper is to show that (2.1)-(2.4) can be reduced to two coupled fourth-order equations for $F$ and $G$ for all minimal midsurfaces. To this end it is convenient, initially, to work with the asymptotic coordinates $u=u^{1}$ and $v=u^{2}$, chosen so that the covariant components of the metric and curvature tensors take the form

$$
\left[a_{\alpha \beta}\right]=P^{2}\left[\begin{array}{cc}
1 & 0 \\
0 & 1
\end{array}\right], \quad\left[b_{\alpha \beta}\right]=\left[\begin{array}{ll}
0 & 1 \\
1 & 0
\end{array}\right],
$$

where $P=(-K)^{-1 / 4}>0$. The function $P$ is not completely arbitrary, but must be such that the Codazzi and Gauss integrability conditions of surface theory are satisfied. It follows from (2.8) that the former equations are satisfied identically, while the latter takes the form [5, Eq. (3-7)]

$$
\left(P^{-1} P_{u}\right)_{u}+\left(P^{-1} P_{v}\right)_{v}=P^{-2}
$$

where a subscript denotes partial differentiation. The operators $L, W$, and $\nabla^{2}$ can be expressed as

$$
\begin{aligned}
L(F) & =P^{-2}\left[\left(P^{2} F_{u}\right)_{u}-\left(P^{2} F_{v}\right)_{v}\right] \\
W(F) & =P^{-2}\left[\left(P^{2} F_{v}\right)_{u}+\left(P^{2} F_{u}\right)_{v}\right] \\
\nabla^{2}(F) & =P^{-2}\left(F_{u u}+F_{v v}\right) .
\end{aligned}
$$

It is also advantageous to introduce new dependent variables and operators as follows: 


$$
\begin{aligned}
(\phi, \gamma, \sigma, \tau) & =P(F, G, S, T) \\
\mathcal{L}(\phi) & \equiv P L\left(P^{-1} \phi\right) \\
& =\phi_{u u}-\phi_{v v}-\left(P_{u u}-P_{v v}\right) P^{-1} \phi \\
\mathscr{W}(\phi) & \equiv P W\left(P^{-1} \phi\right) \\
& =2\left(\phi_{u v}-P_{u v} P^{-1} \phi\right), \\
D(\phi) & \equiv P \nabla^{2}\left(P^{-1} \phi\right)-2 K \phi \\
& =\nabla^{2} \phi-2 P^{-3}\left(P_{u} \phi_{u}+P_{v} \phi_{v}\right)+\left(\nabla^{2} P\right) P^{-1} \phi .
\end{aligned}
$$

In deriving (2.16), we have made use of (2.9).

The governing shell equations (2.1)-(2.4) can now be expressed as

$$
\begin{gathered}
\sigma=\mathscr{L}(\phi)-D W(\tau), \\
\tau=\mathscr{L}(\gamma)+A W(\sigma), \\
W(\phi)+D \mathscr{L}(\tau)+D(1-\mu) D(\gamma)=0, \\
W(\gamma)-A \mathscr{L}(\sigma)-A(1+\nu) D(\phi)=0 .
\end{gathered}
$$

3. The condition of commutability for reduction. Applying $\mathscr{L}$ to (2.18), inserting the resulting right-hand side into (2.19), and solving $(2.20)$ for $\mathcal{L}(\sigma)$, we obtain

$$
\begin{aligned}
W(\phi)+D\left[\mathscr{L}^{2}(\gamma)+W^{2}(\gamma)-A(1+\nu) W D(\phi)+\right. & (1-\mu) D(\gamma)] \\
& +D A(\mathscr{W}-W \mathscr{L})(\sigma)=0 .
\end{aligned}
$$

Likewise, from (2.17), (2.19), and (2.20), we obtain

$$
\begin{aligned}
W(\gamma)-A\left[\mathcal{L}^{2}(\phi)+W^{2}(\phi)+D(1-\mu) W D(\gamma)+\right. & (1+\nu) D(\phi)] \\
& +A D(\mathcal{W}-W \mathcal{L}(\tau)=0 .
\end{aligned}
$$

These are the two coupled fourth-order equations we desire, provided

$$
£ W-W \mathscr{L}=0 .
$$

To express (3.3) in a more explicit yet compact form, define the complex-valued operator

$$
4 \mathfrak{T}=\mathfrak{L}+i w,
$$

and note that

$$
16 \mathfrak{T} \overline{\mathscr{T}}=\mathscr{L}^{2}+W^{2}+i(W \mathcal{L}-\mathscr{L} W) .
$$

Hence $\mathscr{L}$ and $W$ commute if and only if $\mathscr{T} \overline{\mathfrak{T}}$ is a real operator. But in terms of the complex variable

$$
w=u+i v,
$$

(2.14) and (2.15) combine, as indicated by (3.4), to yield

$$
\mathfrak{N}=\left(\partial^{2} / \partial \bar{w}^{2}\right)-\left(P_{\bar{w} \bar{w}} / P\right) .
$$


Since $P$ is real, we have

$$
\begin{aligned}
\operatorname{Tr} T(T) & =\left(\frac{\partial^{2}}{\partial \bar{w}^{2}}-\frac{P_{\bar{w} \bar{w}}}{P}\right)\left(\frac{\partial^{2}}{\partial w^{2}}-\frac{P_{w w}}{P}\right)(\sigma) \\
= & \sigma_{w w \bar{w} \bar{w}}-P^{-1}\left(P_{w w} \sigma_{\bar{w} \bar{w}}+P_{\bar{w} \bar{w}} \sigma_{w w}\right) \\
& =-2\left(P^{-1} P_{\underline{w} \underline{w}}\right)_{\bar{w}} \sigma_{\overline{\bar{w}}}+\left[P^{-2} P_{\bar{w} \bar{w}} P_{w w}-\left(P^{-1} P_{\underline{w} w}\right)_{\bar{w} \bar{w}}\right] \sigma .
\end{aligned}
$$

All terms except those underlined are obviously real. As $\sigma$ is an arbitrary real function of $w$ and $\bar{w}, \sigma_{\bar{w}}$ will, in general, have a non-zero imaginary part. Hence, the first underlined term in (3.8) is guaranteed to be real if and only if

$$
P^{-1} P_{w w}=f(w),
$$

where $f$ is an analytic function of $w$. Condition (3.9) also eliminates the second underlined term in (3.8).

4. Identical satisfaction of the commutability condition. We shall now show that (3.9) is satisfied identically for all analytic, minimal midsurfaces.

It is well known that such surfaces can be expressed in the form [5, p. 188]

$$
\begin{aligned}
& x=\Re \int\left(1-\zeta^{2}\right) g(\zeta) d \zeta, \\
& y=\Re \int i\left(1+\zeta^{2}\right) g(\zeta) d \zeta, \\
& z=\Re \int 2 \zeta g(\zeta) d \zeta,
\end{aligned}
$$

where $g$ is an analytic function of the complex variable $\zeta$, and $R$ denotes "the real part of." The first and second fundamental forms of the surfaces defined by (4.1)-(4.3) work out to be

$$
\begin{aligned}
\mathrm{I} & =4(1+\zeta \bar{\zeta})^{2} g \bar{g} d \zeta d \bar{\zeta}, \\
\mathrm{II} & =2\left(g d \zeta^{2}+\bar{g} d \bar{\zeta}^{2}\right) .
\end{aligned}
$$

The corresponding first and second fundamental forms which follow from (2.8) and (3.6) are

$$
\begin{aligned}
\mathrm{I} & =P^{2} d w d \bar{w}, \\
\mathrm{II} & =-\frac{1}{2} i\left(d w^{2}-d \bar{w}^{2}\right) .
\end{aligned}
$$

These forms can be brought into agreement by setting

$$
\begin{gathered}
P=(1+\zeta \bar{\zeta})(g \bar{g})^{1 / 4}, \\
w_{\zeta}=2 g^{1 / 2} \exp (i \pi / 4) .
\end{gathered}
$$

(The complex $\zeta$-plane is assumed to be appropriately cut so that $g^{1 / 2}$ is analytic there.)

Since (4.4) and (4.5) were derived from (4.1)-(4.3), it must follow that the Gauss integrability condition (2.9), when expressed in terms of $g$ and $\zeta$ via (3.6), (4.8), and (4.9), is satisfied identically. It remains only to verify (3.9). 
By straightforward calculation we have

$$
\begin{aligned}
\frac{1}{P} \frac{d^{2} P}{d w^{2}} & =\frac{1}{(1+\zeta \bar{\zeta}) g^{1 / 4} w_{\zeta}} \frac{d}{d \zeta}\left\{\frac{1}{w_{\zeta}} \frac{d}{d \zeta}\left[(1+\zeta \bar{\zeta}) g^{1 / 4}\right]\right\} \\
& =-\frac{i}{4(1+\zeta \bar{\zeta}) g^{3 / 4}}\left\{\frac{d}{d \zeta}\left[\frac{\bar{\zeta}}{g^{1 / 4}}+\frac{(1+\zeta \bar{\zeta}) g^{\prime}}{4 g^{5 / 4}}\right]\right\} \\
& =\frac{i}{64 g^{3}}\left(5{g^{\prime 2}}^{2}-4 g g^{\prime \prime}\right)
\end{aligned}
$$

which, via (4.9), is an analytic function of $w$, excluding any isolated zeros of $g$.

\section{REFERENCES}

[1] J. L. Sanders, Jr., On the shell equations in complex form, in Proc. Second Sympos. Thin Elastic Shells, Springer-Verlag, Berlin and New York, 1969, pp. 135-156

[2] F. Y. M. Wan, The exact reduction of equations of elastic shells of revolution, Studies in Appl. Math. 48, 361-375 (1969)

[3] J. G. Simmonds, Simplification and reduction of the Sanders-Koiter linear shell equations for various midsurface geometries, Quart. Appl. Math. 28, 259-275 (1970)

[4] J. G. Simmonds, Extension of Koiter's $L_{2}$-error estimate to approximate shell solutions with no strain energy functional, Z. A. M. P. 22, 339-345 (1971)

[5] D. J. Struik, Differential geometry, 2nd edition, Addison-Wesley, Reading, Mass., 1961 